Zbl Arbeitsmed 2016 $66: 337-344$ DOI 10.1007/s40664-016-0118-4 Online publiziert: 27. Juni 2016 (C) The Author(s) 2016. This article is available at SpringerLink with Open Access CrossMark

\author{
P. Morfeld ${ }^{1,2} \cdot$ M. Spallek ${ }^{3,4}$ \\ ${ }^{1}$ Institut und Poliklinik für Arbeitsmedizin, Umweltmedizin und Präventionsforschung, Universität zu Köln, \\ Köln, Deutschland \\ ${ }^{2}$ Institut für Epidemiologie und Risikobewertung in der Arbeitswelt (IERA), Evonik Industries AG, Essen, \\ Deutschland \\ ${ }^{3}$ Institut für Arbeitsmedizin, Sozialmedizin und Umweltmedizin, Goethe-Universität Frankfurt/Main, \\ Frankfurt/Main, Deutschland \\ ${ }^{4}$ Europäische Forschungsvereinigung für Umwelt und Gesundheit im Transportsektor (EUGT e. V.), Berlin, \\ Deutschland
}

\section{Gefährdungsbeurteilung bei Exposition gegenüber mehreren krebserzeugenden Arbeitsstoffen}

\section{Ergänzungen und Erweiterungen}

In einem Beitrag im Zentralblatt für Arbeitsmedizin, Arbeitsschutz und Ergonomie [8] untersuchten wir die Gültigkeit von einfachen Summenformeln zur Gefährdungsbeurteilung bei Exposition gegenüber mehreren (krebserzeugenden) Arbeitsstoffen unter der Annahme binärer Expositionen und eines deterministischen binären Respons. Wir zeigten, dass Exzessrisiken selbst bei Ausschluss präventiver Wirkungen unteradditiv sein können, also notwendigerweise weder eine Additivität noch eine Überadditivität der Exzessrisiken gilt. Daraus folgt, dass einfache Summenformeln keine Rechtfertigung haben, auch nicht zur Abschätzung des Exzessrisikos von unten, da Summenformeln die Additivität behaupten. Das Umweltbundesamt organisierte ein externes Review zu unserer Arbeit, wofür wir uns herzlich bedanken. Die 3 hinzugezogenen Gutachter sind anerkannte Experten für Kausaltheorie und lehren und forschen in Philosophie an deutschen oder Schweizer Universitäten (Prof. Baumgartner, Genf; Prof. Bartels, Bonn; Prof. Beisbart, Bern). Wir möchten uns bei ihnen für den Aufwand und die Akribie bedanken, mit der unser Beitrag kritisch gelesen und bewertet wurde.

Von 2 Gutachtern wurde zu Recht bemängelt, dass in dem dritten erläuternden Beispiel zur Tab. 2 auf S. 196 in [8] nicht der Responstyp 15, sondern der Typ 9 beschrieben wird. Herr Konietzka, Umweltbundesamt, informierte uns zudem, dass es in der Überschrift zu Tab. 1 auf S. 196 in [8] nicht „Y“, sondern „Z“ "heißen muss. Wir danken den Reviewern und Herrn Konietzka für die Aufdeckung der Tippfehler.

Die Gutachter unterstützen unsere Hauptaussage, dass die vorgeschlagenen einfachen Summenformeln keine allgemeine Gültigkeit haben können. Wichtiger ist jedoch, dass die Reviewer der Publikation wesentliche ergänzende Fragen aufwarfen, die in unserem Beitrag nicht bearbeitet sind. Wir halten diese Fragen für so bedeutend, dass wir sie mit dieser Ergänzung zum Originalbeitrag der wissenschaftlichen Öffentlichkeit vorstellen möchten.

Die Fragen:

1. Sind Exzesswahrscheinlichkeiten Wahrscheinlichkeiten im strikt mathematischen Sinn, d. h., genügen Exzesswahrscheinlichkeiten den Kolmogorov-Axiomen? Wenn dem so ist, können Exzesswahrscheinlichkeiten nicht negativ werden, was insbesondere Gl. (8) in [8] problematisiert, die genau dies behauptet. Wesentliche Folgerungen in [8] hängen an der Gültigkeit von Gl. (8).

2. Warum werden präventive Wirkungen im wesentlichen Teil der Arbeit ausgeschlossen? Zumindest theoretisch können auch präventive Wirkungen auftreten. Zudem haben Morfeld und Spallek [8] den Begriff "präventiv" sehr weit gefasst und damit auch Responstypen ausgeschlossen, die in Einzelsituationen adverse Wirkungen zeigen. Was ergibt sich, wenn die Auswertung auf Responstypen eingeschränkt wird, die in einem weiten oder auch engen Sinne adverse Wirkungen beinhalten?

3. Die Arbeit setzt 2 binäre Expositionsvariablen voraus. Was ergibt sich, wenn intervallskalierte Expositionen zugelassen werden?

4. Die Arbeit setzt einen deterministischen binären Respons voraus. Wie ändern sich die Hauptergebnisse, wenn ein probabilistischer Respons angenommen wird?

Einige dieser Fragen wirken auf den ersten Blick technisch, haben aber eine große Tragweite. Die Fragen 1) und 2) bezweifeln die Belastbarkeit der Aussagen in Morfeld und Spallek [8]. Die Fragen 3) und 4) hingegen zielen darauf, dass die veröffentlichte Arbeit keine hinreichende Allgemeingültigkeit beanspruchen kann, um praxisrelevante Situationen von höherer Komplexität einzuschließen.

Im Folgenden stellen wir uns diesen 4 Fragenkomplexen. 


\section{Exzessrisiken sind keine Wahrscheinlichkeiten}

Die von uns in [8] berechneten Risiken sind Wahrscheinlichkeiten im Sinne der Kolmogorov-Axiome. Risiken sind deshalb notwendigerweise nicht-negativ. Exzessrisiken sind aber keine Wahrscheinlichkeiten, auch nicht Differenzen zwischen Exzessrisiken. Der Begriff „Exzessrisiko“ impliziert also nicht, dass Exzessrisiken oder Differenzen zwischen Exzessrisiken notwendig $\geq 0$ sind.

\section{Begründung}

Die aus Tab. 2 und 3 in [8] abgeleiteten Risiken berechnen sich als Summen über die relativen Häufigkeiten disjunkter Responstypen. Da relative Häufigkeiten zu endlichen Mengen Wahrscheinlichkeiten im Sinne der Kolmogorov-Axiome darstellen ${ }^{1}$, können wir das Additionsaxiom anwenden, das die Additivität (allgemeiner: $\sigma$-Additivität) der Wahrscheinlichkeiten disjunkter Ereignisse sichert. Damit ergeben sich die Risiken $R_{00}, R_{01}, R_{10}$ und $R_{11}$ als Wahrscheinlichkeiten. Der Klarheit halber sei ergänzt, dass wir von bedingten Wahrscheinlichkeiten sprechen, die über verschiedenen Wahrscheinlichkeitsräumen definiert sind und die sich aufgrund der Bedingungen $(00,01,10,11)$ unterscheiden. Daher addieren sich diese bedingten Risiken $R_{00}, R_{01}, R_{10}$ und $R_{11}$ nicht notwendig $\mathrm{zu}$ 1. Exzessrisiken sind Ausdrücke einer höheren Stufe und werden nicht über Operationen in einer zugrunde liegenden Mengenalgebra definiert, sondern durch direkte Differenzbildung zwischen den bedingten Wahrscheinlichkeiten aus unterschiedlichen Wahrscheinlichkeitsräumen, z. B. $R_{01}-R_{00}$ (s. im Glossar 10.1 der TRGS 910 [6] die Definition der Begriffe „Exzessrisiko“ (b) auf S. 114 bzw. gleichbedeutend "Additional Risk“ auf S. 103). Wahrscheinlichkeiten haben schwache algebraische Eigenschaften.

\footnotetext{
1 Die Teilmengen einer endlichen Menge bilden mit den Operationen Durchschnitt und Vereinigung eine $\sigma$-Algebra, und die 3 Kolmogorov-Axiome gelten für relative Häufigkeiten. Zu den verwendeten Begriffen aus der Wahrscheinlichkeitstheorie sei auf Lehrbücher verwiesen, z. B. [3] und [7].
}

Es existiert ein neutrales Element der Addition und der Multiplikation, aber es gibt keine Inversen (sie müssten kleiner als 0 bzw. größer als 1 sein, was der Normierung widerspricht). Deshalb muss die Differenz zweier Wahrscheinlichkeiten nicht notwendigerweise eine Wahrscheinlichkeit darstellen. Dies gilt schon gar nicht für die Differenz bedingter Wahrscheinlichkeiten, die nicht über demselben Wahrscheinlichkeitsraum definiert sind. $R_{00}-R_{01}$ ist somit im Allgemeinen keine Wahrscheinlichkeit, und sicherlich ist es das nicht, sobald $R_{00}<R_{01}$. Dasselbe gilt für noch weiter gehende Verrechnungen wie Differenzen von Wahrscheinlichkeitsdifferenzen. Insofern ist es ein Fehlschluss anzunehmen, dass $R_{11}-R_{00}-\left(R_{10}-R_{00}\right)-\left(R_{01}-R_{00}\right)$ notwendig nicht-negativ ist, denn dieser Ausdruck ist keine Wahrscheinlichkeit im Sinne der Kolmogorov-Axiome. Wir bestreiten nicht, dass es Situationen geben kann, in denen $R_{11}-R_{00}=\left(R_{10}-\right.$ $\left.R_{00}\right)+\left(R_{01}-R_{00}\right)$ oder $R_{11}-R_{00}>\left(R_{10}-\right.$ $\left.R_{00}\right)+\left(R_{01}-R_{00}\right)$ gelten mag. Unsere Argumentation beruht allein auf der Aussage, dass $R_{11}-R_{00}<\left(R_{10}-R_{00}\right)+$ $\left(R_{01}-R_{00}\right)$ nicht ausgeschlossen werden kann, da $R_{11}-R_{00}-\left(R_{10}-R_{00}\right)-\left(R_{01}-\right.$ $\left.R_{00}\right)$ keine Wahrscheinlichkeit ist.

Die allgemeine Sprachregelung mag unglücklich und irritierend sein, Ausdrücke wie $R_{11}-R_{00}$ oder $R_{11}-R_{00}$ $\left(R_{10}-R_{00}\right)-\left(R_{01}-R_{00}\right)$ als ,Exzessrisiken“" zu bezeichnen, denn diese Terme stellen keine Risiken im Sinne von Wahrscheinlichkeiten dar. Für die aktuellen Diskussionen ist es deshalb umso wichtiger, die mathematischen Eigenschaften dieser Ausdrücke klar vor Augen zu haben. Alle Versuche, einfache Summenformeln dadurch zu rechtfertigen, dass man Exzessrisiken als Wahrscheinlichkeiten behandelt, müssen daher scheitern, obwohl solche Argumentationen vordergründig plausibel sein mögen.

\section{Adverse Reaktionstypen}

Die Einschränkung auf adverse Reaktionstypen (in einem weiten oder in einem engen Sinn gefasst) erlaubt viele Konstellationen, in denen die Exzessrisiken unteradditiv sind. Einfache Summenformeln gelten also auch in dieser Situation nicht. Dieses Ergebnis überrascht nicht, da in Morfeld und Spallek [8] gezeigt wurde, dass selbst bei Ausschluss aller präventiven Expositionswirkungen eine Unteradditivität der Exzessrisiken auftreten kann.

\section{Begründung}

Der Fragenkomplex 2 enthält 2 voneinander logisch unabhängige Teilfragen:

2a) Warum haben die Autoren in [8] die Betrachtung unter der Bedingung durchgeführt, dass präventive Wirkungen ausgeschlossen werden?

2b) Was ergibt sich, wenn stattdessen die Betrachtung auf adverse Wirkungen eingeschränkt wird?

Bevor wir die Fragen beantworten, möchten wir klarstellen, dass unsere zentrale Aussage, dass weder die Additivität noch die Überadditivität der Exzessrisiken folgt und deshalb einfache Summenformeln keine Rechtfertigung haben, bereits ohne jede zusätzliche Bedingung gilt (s. hierzu die Gl. (9) in [8] und die zugehörigen Diskussionen). Es stellt sich somit lediglich die Frage, ob denn die einfachen Formeln zu "retten“ sind, wenn einschränkende Bedingungen auferlegt werden. Wir betonen noch einmal, dass klar ist, dass die einfachen Summenformeln ohne die Einführung solcher besonderen Bedingungen nicht gelten. In [8] untersuchten wir, ob eine „Rettung“ der einfachen Formeln möglich wird, wenn präventive Wirkungen a priori ausgeschlossen werden (entspricht Frage 2a, die mit „nein“ $z u$ beantworten ist). Jetzt untersuchen wir zusätzlich, ob dies denn gelingen kann, wenn die Betrachtung auf adverse Wirkungen eingeschränkt wird (entspricht Frage 2b).

Zunächst zur Teilfrage 2a: Wir haben den Begriff der präventiven Wirkung in [8] weit gefasst. Es gelten alle Reaktionstypen als präventiv, die mindestens eine präventive Wirkung beinhalten. Wir zählen damit 10 Reaktionstypen als präventiv, bei denen entweder eine Einzelexposition oder die Doppelexposition den Wert von $Y$ im Vergleich zum Basiswert oder zum Wert einer Einzelexposition auf 0 setzt (s. Tab. 2 in [8]). Die Reaktionsmuster der Responstypen 10, 
Hier steht eine Anzeige.

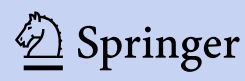


12 und 14 weisen alle einen Basiswert von $Y=0$ auf und zeigen bei Einzelexpositionen $Y=1$. Dies bedeutet, hier haben die Expositionen $X$ oder $Z$ adverse Wirkung. Trotzdem wurden diese Reaktionsmuster in [8] als „präventiv“ gewertet, weil bei Doppelexposition der Wert von $Y$ wieder 0 ist. Wir haben bewusst diesen weiten Begriff des präventiven Responstyps gewählt, um in [8] zu zeigen, dass selbst bei strengstem Ausschluss präventiver Wirkungen eine Unteradditivität von Exzessrisiken auftreten kann. Die Diskussion ist zwar theoretisch offen für präventive Wirkungen - so ist es durchaus möglich, dass sich 2 Kanzerogene bei gleichzeitiger Exposition gegenseitig blockieren - jedoch wird in einer Abhandlung der Deutschen Gesellschaft für Arbeits- und Umweltmedizin diesem Phänomen nicht viel Raum und Gewicht gegeben [10, s. auch: http://www.dgaum. de/fileadmin/PDF/Stellungnahmen_ Positionspapiere/Positionspapier_ Synkanzerogenese.pdf]. In einer juristischen Stellungnahme führte dies zum Missverständnis, dass sich die „Einwirkungen wechselseitig verstärken und zumindest (!) additiv wirken “ ([5], s. auch den zugehörigen Leserbriefwechsel in $[4,9])$. Morfeld und Spallek [8] zeigten, dass auch unter der Annahme des Ausschlusses präventiver Wirkungen weder die Additivität noch die Überadditivität der Exzessrisiken folgt und deshalb einfache Summenformeln selbst in diesem strikten Szenario keine Rechtfertigung haben, auch nicht zur Abschätzung der Exzessrisiken von unten. Dies gilt natürlich aber insbesondere dann, wenn präventive Wirkungen zugelassen werden, wie wir im Folgenden beispielhaft für einen weit gefassten Begriff der adversen Wirkung darlegen. Wir untersuchen zusätzlich auch einen eng gefassten Begriff der adversen Wirkung, um die Frage $2 b$ möglichst vollständig zu bearbeiten.

Nun zur Teilfrage 2b: Wir fassen ,adverse Wirkung“ zunächst ähnlich weit wie den Begriff der „präventiven Wirkung" und definieren Reaktionstypen in Tab. 2 [8] als "advers“, die mindestens eine nachteilige Wirkungskomponente aufweisen: 2, 3, 4, 5, 6, 7, 8, 10, 12 und 14. Diese können wir gemeinsam untersuchen, indem $p_{1}=0, p_{9}=0, p_{11}=0$,

Zbl Arbeitsmed 2016 -66:337-344 DOI 10.1007/s40664-016-0118-4

(c) The Author(s) 2016 . This article is available at SpringerLink with Open Access

P. Morfeld · M. Spallek

\section{Gefährdungsbeurteilung bei Exposition gegenüber mehreren krebserzeugenden Arbeitsstoffen. Ergänzungen und Erweiterungen}

\section{Zusammenfassung}

Wir untersuchten die Gültigkeit von einfachen Summenformeln zur Gefährdungsbeurteilung bei Exposition gegenüber mehreren (krebserzeugenden) Arbeitsstoffen unter der Annahme binärer Expositionen und eines deterministischen Respons. Das Umweltbundesamt organisierte ein externes Review unserer Arbeit. Die Gutachter unterstützen unsere Hauptaussage, dass Summenformeln keine allgemeine Gültigkeit haben können, haben aber ergänzende Fragen gestellt. In diesem Nachtrag möchten wir die wissenschaftliche Öffentlichkeit darüber informieren und auf die gestellten Fragen eingehen. Wir zeigen, dass die wesentlichen Aussagen im oben genannten
Beitrag gültig bleiben, auch wenn die Expositionen intervallskaliert sind oder der binäre Respons probabilistisch ist oder die Analysen auf Responstypen eingeschränkt werden, die adverse Effekte beschreiben. Wir stellen zudem klar, dass Exzessrisiken und Differenzen von Exzessrisiken keine Wahrscheinlichkeiten im Sinne der KolmogorovAxiome darstellen. Einfache Summenformeln haben damit keine Rechtfertigung, auch nicht zur Abschätzung des Exzessrisikos von unten.

\section{Schlüsselwörter}

Synergie · Kanzerogenität · Probabilistischer Respons · Kolmogorov-Axiome · Summenformel

\section{Hazard assessment by exposure to several carcinogenic working materials. Supplements and enhancements}

\begin{abstract}
We analyzed the validity of simple summation formulae with which a common effect of different occupational exposures could be mapped to the same cancer endpoint. Our paper published in this journal relied on the assumptions of a deterministic response and binary exposures. The German Federal Environmental Agency organized an external review of our paper. Although the reviewers agreed with our main conclusion that simple summation formulae cannot have a general validity, they raised questions that we would like to inform the scientific public about and are addressed in this addendum. We demonstrate that the main statements in
\end{abstract}

Morfeld and Spallek 2015 remain valid even if the exposures are defined on an interval scale, the binary response is probabilistic or if the analyzed response types are restricted to adverse types. We clarify that excess risks and differences in excess risks are not probabilities according to the Kolmogorov axioms. Simple summation formulae are therefore not justified, not even to estimate the lower bounds for excess risks.

Keywords

Synergy · Carcinogenicity · Probabilistic response $\cdot$ Kolmogorov axioms $\cdot$ Sum formula $p_{13}=0, p_{15}=0$ und $p_{16}=0$ gesetzt werden . Wir betonen, dass die Responstypen 3, $5,7,10,12$ und 14 gleichzeitig präventiv sind (im obigen weiten Sinn). Die beiden untersuchten Bedingungen (Teilfrage 2a, Teilfrage $2 b$ ) stehen somit nicht in einem logischen Abhängigkeitsverhältnis.

Aus Gl. (9) in [8] ergibt sich für die adversen Reaktionstypen:

$$
\begin{aligned}
R_{11}-R_{00}- & \left(R_{10}-R_{00}\right) \\
& -\left(R_{01}-R_{00}\right)= \\
=- & p_{2}+p_{3}+p_{5}+2 p_{7}+p_{8} \\
- & p_{9}-2 p_{10}-p_{12}-p_{14}+p_{15} \\
= & -p_{2}+p_{3}+p_{5}+2 p_{7}+p_{8} \\
- & 2 p_{10}-p_{12}-p_{14} .
\end{aligned}
$$


Dieser Ausdruck wird negativ genau dann, wenn $p_{3}+p_{5}+2 p_{7}+p_{8}<p_{2}+$ $2 p_{10}+p_{12}+p_{14}$.

Auch eine Einschränkung auf adverse Wirkungen kann somit nicht ausschließen, dass viele Konstellationen auftreten, in denen die Exzessrisiken unteradditiv sind. Dies gilt auch dann, wenn zusätzlich die gleichzeitig als präventiv eingestuften Typen 3, 5, 7, 10, 12 und 14 eliminiert werden, also eine enge Fassung des Begriffs „advers“ erfolgt. Die obige Gleichung reduziert sich in dieser Situation $\mathrm{zu}$

$$
\begin{array}{r}
R_{11}-R_{00}-\left(R_{10}-R_{00}\right) \\
-\left(R_{01}-R_{00}\right)=-p_{2}+p_{8} .
\end{array}
$$

Dieser Ausdruck wird negativ genau dann, wenn $p_{8}<p_{2}$, was stets möglich ist.

Dieses Ergebnis zu adversen Wirkungen (im weiten wie im engen Sinne) überrascht nicht, da in [8] gezeigt wurde, dass selbst bei Ausschluss aller präventiven Expositionswirkungen eine Unteradditivität der Exzessrisiken auftreten kann, also die einfachen Summenformeln weder eine unverzerrte Schätzung des Exzessrisikos erlauben noch dessen Abschätzung von unten.

\section{Intervallskalierte Expositions- variablen}

Auch bei intervallskalierten Expositionsvariablen $X$ und $Z$ kann durchgängig eine Unteradditivität der Exzessrisiken vorliegen. Dies gilt selbst dann, wenn $X$ und $Z$ nie präventiv wirken. Die in Morfeld und Spallek [8] für binäre Expositionen gezeigte Aussage, dass einfache Summenformeln selbst bei Ausschluss präventiver Wirkungen keine Rechtfertigung haben, auch nicht zur Abschätzung der Exzessrisiken von unten, gilt ebenfalls für intervallskalierte Variablen.

\section{Begründung}

Wir zeigen dies im Folgenden für beliebige, ansteigende Expositions-RisikoBeziehungen der Expositionen $X$ und $Z$ und beginnen mit der Festlegung entsprechender Funktionen: $f_{1}(X)$ und $f_{2}(Z)$ seien streng monoton steigende Funktionen nach $[0,1)$, definiert über den reellen
Intervallen $\left\{X \mid 0 \leq X \leq X_{\max }\right\}$ bzw. $\{Z \mid 0$ $\left.\leq Z \leq Z_{\max }\right\}$, wobei für die Ausgangswerte

$$
f_{1}(0)=f_{2}(0)=c, \quad 0 \leq c<1
$$

gelten soll. $X_{\max }$ und $Z_{\max }$ können unendlich sein. Wir benutzen diese beiden Funktionen $f_{1}$ und $f_{2}$ weiter unten, um die bedingten Wahrscheinlichkeiten $P(Y=1$ | $X, Z)$ zu definieren. Jedoch führen wir zunächst eine gemeinsame Funktion $f$ ein.

Die gemeinsame Funktion $f$ wird mithilfe von $f_{1}$ und $f_{2}$ wie folgt über $\{X, Z \mid$ $\left.0 \leq X \leq X_{\max }, 0 \leq Z \leq Z_{\max }\right\}$ definiert:

$$
\begin{aligned}
f(X, Z)= & f_{1}(X)+f_{2}(Z) \\
& -\left(f_{1}(X)-c\right) \\
& \cdot\left(f_{2}(Z)-c\right)-c .
\end{aligned}
$$

Es gilt dann

$$
\begin{aligned}
& f(X, 0)=f_{1}(X) \text { und } \\
& f(0, Z)=f_{2}(Z) .
\end{aligned}
$$

Des Weiteren folgt für $X>0$ und $Z$ $>0$ eine doppelte Ungleichung:

$$
\begin{aligned}
0 & <\left(f_{1}(X)-c\right) \cdot\left(f_{2}(Z)-c\right) \\
& <\min \left(f_{1}(X), f_{2}(Z)\right) .
\end{aligned}
$$

Die erste Ungleichung gilt, da $f_{1}(X)-c$ und $f_{2}(Z)-c$ beide positiv sind. Die zweite gilt, da $f_{1}(X)-c<1$ und $f_{2}(Z)-c<1$ und $f_{1}(X)-c \leq f_{1}(X)$ und $f_{2}(Z)-c \leq f_{2}(Z)$ : $\left(f_{1}(X)-c\right) \cdot\left(f_{2}(Z)-c\right)<1 \cdot\left(f_{2}(Z)-c\right) \leq f_{2}(Z)$ und $\left(f_{1}(X)-c\right) \cdot\left(f_{2}(Z)-c\right)<\left(f_{1}(Z)-c\right) \cdot 1$ $\leq f_{1}(Z)$, also zusammengefasst

$$
\begin{aligned}
& \left(f_{1}(X)-c\right) \cdot\left(f_{2}(Z)-c\right) \\
& <\min \left(f_{1}(X), f_{2}(Z)\right) .
\end{aligned}
$$

Somit ist z. B. klar, dass $f(X, Z)>f(0, Z)$ für $X>0$ und $f(X, Z)>f(X, 0)$ für $Z>0$.

Wichtig für unsere folgenden Anwendungen ist die allgemeinere Aussage:

$$
f_{1}(X)-\left(f_{1}(X)-c\right) \cdot\left(f_{2}(Z)-c\right)
$$

ist streng monoton steigend in $X$ für jedes feste $Z$. Denn

$$
\begin{aligned}
& f_{1}(X)-\left(f_{1}(X)-c\right) \cdot\left(f_{2}(Z)-c\right) \\
& =(1-g) \cdot f_{1}(X)+g \cdot c, \\
& 0 \leq g:=f_{2}(Z)-c<1,
\end{aligned}
$$

und $f_{1}(X)$ ist streng monoton steigend. Entsprechend folgt,

$$
f_{2}(Z)-\left(f_{1}(X)-c\right) \cdot\left(f_{2}(Z)-c\right)
$$

ist streng monoton steigend in $Z$ für jedes feste $X$.

Mithilfe von $f$ wird die bedingte Wahrscheinlichkeit festgesetzt, dass der binäre Respons $Y \in\{0,1\}$ den Wert 1 annimmt, also das Risiko für $Y=1$ bei $X$ und $Z$ :

$$
R_{X Z}=P(Y=1 \mid X, Z)=f(X, Z) .
$$

Damit gilt aufgrund der Eigenschaften von $f_{1}, f_{2}$ und $f$, dass die Auftrittsrisiken für $Y=1$ immer streng monoton über den Expositionen steigen. Es gibt also keine präventiven Wirkungen, d. h., nirgendwo im Definitionsbereich $\left\{X, Z \mid 0 \leq X \leq X_{\max }\right.$, $\left.0 \leq Z \leq Z_{\text {max }}\right\}$ gibt es eine Risikoreduktion durch Expositionssteigerung.

Als bedingte Risiken für $Y=1$ berechnen sich:

- Basisrisiko:

$$
\begin{aligned}
R_{00}= & f_{1}(0)+f_{2}(0)-\left(f_{1}(0)-c\right) \\
& \cdot\left(f_{2}(0)-c\right)-c=c,
\end{aligned}
$$

- Risiko bei $X$ beliebig und $Z=0$ :

$$
\begin{aligned}
R_{X 0}= & f_{1}(X)+f_{2}(0) \\
& -\left(f_{1}(X)-c\right) \cdot\left(f_{2}(0)-c\right)-c \\
= & f_{1}(X),
\end{aligned}
$$

- Risiko bei $Z$ beliebig und $X=0$ :

$$
\begin{aligned}
R_{0 Z}= & f_{1}(0)+f_{2}(Z) \\
& -\left(f_{1}(0)-c\right) \cdot\left(f_{2}(Z)-c\right)-c \\
= & f_{2}(Z),
\end{aligned}
$$

- Risiko bei $X$ beliebig und $Z$ beliebig:

$$
\begin{aligned}
R_{\mathrm{XZ}}= & f_{1}(X)+f_{2}(Z) \\
& -\left(f_{1}(X)-c\right) \cdot\left(f_{2}(Z)-c\right)-c .
\end{aligned}
$$

Für die 3 Exzessrisiken folgt:

$$
\begin{aligned}
R_{X 0}-R_{00}= & f_{1}(X)-c, \\
R_{0 Z}-R_{00}= & f_{2}(Z)-c, \\
R_{X Z}-R_{00}= & f_{1}(X)+f_{2}(Z) \\
& -\left(f_{1}(X)-c\right) \\
& \cdot\left(f_{2}(Z)-c\right)-2 c .
\end{aligned}
$$


Tab. 1 Wahrscheinlichkeit der Beobach-

tung einer vertikalen oder horizontalen

Polarisation an Photonen nach der Passage

eines polarisierenden Strahlteilers, der um

den Winkel $a$ im Uhrzeigersinn gegen die

ursprüngliche Polarisationsebene der Pho-

tonen gedreht ist. Es gilt $\alpha=10^{\circ}+X \cdot 12,5^{\circ}+$

$Z \cdot 35^{\circ}$

\begin{tabular}{|lllll}
\hline $\boldsymbol{X}^{\mathbf{a}}$ & $\boldsymbol{Z}^{\mathbf{a}}$ & $\begin{array}{l}\boldsymbol{a} \\
\left.\text { (in }^{\circ}\right)\end{array}$ & $\begin{array}{l}\boldsymbol{P}(\boldsymbol{Y}=\mathbf{1} \mid \\
\boldsymbol{X}, \boldsymbol{Z})^{\mathbf{b}}\end{array}$ & $\begin{array}{l}\boldsymbol{P}(\boldsymbol{Y}=\mathbf{0} \mid \\
\boldsymbol{X}, \boldsymbol{Z})^{\mathbf{c}}\end{array}$ \\
\hline 0 & 0 & 10 & 0,97 & 0,03 \\
\hline 0 & 1 & 22,5 & 0,85 & 0,15 \\
\hline 1 & 0 & 45 & 0,50 & 0,50 \\
\hline 1 & 1 & 57,5 & 0,29 & 0,71 \\
\hline
\end{tabular}

${ }^{\mathrm{a} B i n a ̈ r e ~ E x p o s i t i o n s v a r i a b l e n . ~}{ }^{\mathrm{b}}$ Bedingte

Wahrscheinlichkeit für vertikale Polarisation.

'Bedingte Wahrscheinlichkeit für horizontale

Polarisation

Damit ergibt sich zur Frage der Additivität der Exzessrisiken:

$$
\begin{aligned}
& R_{X Z}-R_{00}-\left(R_{0 Z}-R_{00}\right) \\
& -\left(R_{X 0}-R_{00}\right) \\
= & -\left(f_{1}(X)-c\right) \cdot\left(f_{2}(Z)-c\right)
\end{aligned}
$$

und deshalb

$$
\begin{aligned}
& R_{X Z}-R_{00}-\left(R_{0 Z}-R_{00}\right) \\
& -\left(R_{X 0}-R_{00}\right)<0, \\
& \text { falls } X>0 \text { und } Z>0 .
\end{aligned}
$$

Also sind die Exzessrisiken immer unteradditiv.

Wir haben gezeigt: Bei intervallskalierten Variablen $X$ und $Z$ kann durchgängig eine Unteradditivität der Exzessrisiken vorliegen. Und diese Aussage zur Unteradditivität gilt selbst dann, wenn jede Expositionserhöhung risikosteigernd wirkt, also $X$ und $Z$ niemals eine präventive Wirkung haben. Wir betonen, dass hier eine bestimmte Klasse von Modellen für die Wahrscheinlichkeit des binären Respons angenommen wird (die Annahme besteht darin, dass die bedingte Wahrscheinlichkeit bei Doppelexposition durch $f(X, Y)$ ausgedrückt wird; das ist nicht der allgemeine Fall). Die Beschränkung auf eine bestimmte Modellklasse ist aber völlig ausreichend, da es darum geht zu zeigen, dass Unteradditivität vorliegen kann. Die Beschränkung verhindert aber eine allgemeine Aussage, dass immer Unteradditivität vorliegt. Wir behaupten auch nicht, dass es keine
Situation geben kann ohne Unteradditivität. Wir haben aber bewiesen, dass eine solche Unteradditivität nie ausgeschlossen werden kann.

Die Argumentation hat dennoch einen recht allgemeinen Charakter, da für $f_{1}$ und $f_{2}$ beliebige, streng monoton steigende Expositions-Risiko-Beziehungen gewählt werden können. Einfache Summenformeln sind nicht zu rechtfertigen, auch nicht zur Abschätzung der Exzessrisiken von unten.

\section{Probabilistischer Respons}

Die Tab. 2 und 3 in Morfeld und Spallek [8] gelten auch bei Annahme eines probabilistischen, d. h. eines indeterministischen Respons. Die aus den Tabellen abgeleiteten Aussagen sind damit auch gültig. Insbesondere gilt die folgende in Morfeld und Spallek [8] für einen deterministischen Respons gezeigte Aussage auch bei probabilistischem Respons: Exzessrisiken können unteradditiv sein selbst dann, wenn keine präventiven Wirkungen vorliegen. Einfache Summenformeln sind auch nicht bei Annahme einer probabilistischen Responsvariablen zu rechtfertigen, selbst dann nicht, wenn sie allein zur Abschätzung des Exzessrisikos von unten verwendet werden sollen.

\section{Begründung}

Morfeld und Spallek [8] arbeiten mit einer deterministischen Responsvariablen. Es kann sicherlich bestritten werden, ob hiermit alle wesentlichen Situationen eingeschlossen werden, da z. B. die Entwicklung von Lungenkrebs nach Exposition gegenüber Radon im untertägigen Uranbergbau indeterministisch ist, weil der Zerfall der eingeatmeten Radonatome den Gesetzen der Quantenmechanik (QM) unterliegt. Im Folgenden soll daher untersucht werden, ob die Berücksichtigung einer strengen Indeterminiertheit von Ereignissen (im Sinne der QM) zu anderen Folgerungen führt. Hierzu betrachten wir das folgende Experiment.

Linear polarisiertes Licht - bestehend aus einzelnen Photonen, präpariert in einem Quantenzustand $\Psi$ mit vertikaler Polarisation - wird durch einen polarisie- renden Strahlteiler geschickt, der um den Winkel $\alpha$ im Uhrzeigersinn gegen die Polarisationsebene der eintretenden Photonen gedreht ist ${ }^{2}$. Der eingestellte Winkel $\alpha$ hänge folgendermaßen von der Kombination zweier binärer Expositionsvariablen $X$ und $Z \mathrm{ab}^{3}$ :

$$
\alpha=10^{\circ}+X \cdot 12,5^{\circ}+Z \cdot 35^{\circ} .
$$

Hinter dem Strahlteiler befinden sich in Richtung der beiden Ausgangsstrahlen Detektoren. Als Respons Y wird verfolgt, ob eine vertikale (1) oder horizontale (0) Polarisation nach Durchlaufen des Strahlteilers an den Detektoren gefunden wird. „Vertikal“ und „horizontal“ werden in Bezug auf den Stahlteiler bestimmt. Ist das Ergebnis $Y=1$ (vertikal), so ist die Polarisationsebene um $\alpha$ gegenüber der Ausgangslage gedreht, bei $Y=0$ (horizontal) um $\alpha-90^{\circ}$. Die Messungen erfolgen zerstörungsfrei, sodass weitere Untersuchungen an dem Photon angestellt werden können, nachdem es den Detektor passiert hat (s. [1], S. 63). Für unsere Überlegungen ist von zentraler Bedeutung: Der Respons $Y$ ist bis zur Messung an den Detektoren in Superposition, also indeterminiert. Jedoch sind die bedingten Wahrscheinlichkeiten für $Y=1$ bzw. $Y=0$ in den Detektoren nach QM auch ohne Messung definit. Die Born-Regeln lassen sich anwenden (vgl. [1], S. 75), und es gilt:

$$
\begin{aligned}
& P(Y=1 \mid X, Z)=\cos ^{2}(\alpha) \quad \text { und } \\
& P(Y=0 \mid X, Z)=\sin ^{2}(\alpha) .
\end{aligned}
$$

Die sich ergebenden Werte sind in - Tab. 1 dargestellt.

Im eigentlichen Experiment wird diese Strahlteiler-Detektor-Kombination auf jedem möglichen Weg der Photonen 4-fach hintereinander aufgebaut, wobei an den Strahlteilern nacheinander

\footnotetext{
2 Dieser Versuchsaufbau ist in [1] auf S. 41, 55f und 71-77 ausführlich dargestellt. Zur detaillierten mathematischen Beschreibung der linearen Polarisation und der Zustandsentwicklung von Photonen an Strahlteilern sei auf Lehrbücherzur QM verwiesen, siehez. B.S.62-66 in [2].

${ }_{3}$ Der Ausdruck kann durch viele andere Funktionen ersetzt werden, ohne dass sich die wesentliche Folgerung aus unseren Überlegungenändert.
} 
Tab. 2 Auftrittswahrscheinlichkeit $P$ der 16 Responstypen im Experiment

Typ $Y(0,0) \quad Y(0,1) \quad Y(1,0) \quad Y(1,1) P$

\begin{tabular}{|llllll}
\hline 1 & 0 & 0 & 0 & 0 & $p_{1}$ \\
\hline 2 & 0 & 0 & 0 & 1 & $p_{2}$ \\
\hline 3 & 0 & 0 & 1 & 0 & $p_{3}$ \\
\hline 4 & 0 & 0 & 1 & 1 & $p_{4}$ \\
\hline 5 & 0 & 1 & 0 & 0 & $p_{5}$ \\
\hline 6 & 0 & 1 & 0 & 1 & $p_{6}$ \\
\hline 7 & 0 & 1 & 1 & 0 & $p_{7}$ \\
\hline 8 & 0 & 1 & 1 & 1 & $p_{8}$ \\
\hline 9 & 1 & 0 & 0 & 0 & $p_{9}$ \\
\hline 10 & 1 & 0 & 0 & 1 & $p_{10}$ \\
\hline 11 & 1 & 0 & 1 & 0 & $p_{11}$ \\
\hline 12 & 1 & 0 & 1 & 1 & $p_{12}$ \\
\hline 13 & 1 & 1 & 0 & 0 & $p_{13}$ \\
\hline 14 & 1 & 1 & 0 & 1 & $p_{14}$ \\
\hline 15 & 1 & 1 & 1 & 0 & $p_{15}$ \\
\hline 16 & 1 & 1 & 1 & 1 & $p_{16}$ \\
\hline
\end{tabular}

die Kombinationen $X=0$ und $Z=0$, $X=0$ und $Z=1, X=1$ und $Z=0$ sowie $X=1$ und $Z=1$ eingestellt werden. Der Winkel $\alpha$ bedeutet stets eine weitere Drehung im Uhrzeigersinn aus der letzten im Detektor bestimmten Polarisationsebene.

Das Experiment hat 16 verschiedene mögliche Ausgänge für die $4 Y$-Variablen $Y(0,0), Y(0,1), Y(1,0)$ und $Y(1,1)$, die an den Photonen vermessen werden. $Y(i, j)$ mit $i$ und $j \in\{0,1\}$, bezeichnet den Messwert ( $Y=1$ : vertikal, $Y=0$ : horizontal), der nach Passage des Strahlteilers, an $\operatorname{dem} X=i$ und $Z=j$ eingestellt sind, vom Detektor registriert wird. Die - Tab. 2 zeigt die 16 möglichen und disjunkten Responskombinationen (Responstypen). Jedes der 16 möglichen Ergebnisse hat eine definite Auftrittswahrscheinlichkeit. Zum Beispiel beträgt die Wahrscheinlichkeit, den Typen $16 \mathrm{zu}$ beobachten, also 4-mal $Y=1: p_{1}=P(Y=1 \mid X=0$, $Z=0) \cdot P(Y=1 \mid X=0, Z=1) \cdot P(Y=1 \mid$ $X=1, Z=0) \cdot P(Y=1 \mid X=1, Z=1)=$ $0,97 \cdot 0,85 \cdot 0,50 \cdot 0,29=0,12$. Für Typ 6 erhält man: $p_{6}=P(Y=0 \mid X=0$, $Z=0) \cdot P(Y=1 \mid X=0, Z=1) \cdot P(Y=0 \mid$ $X=1, Z=0) \cdot P(Y=1 \mid X=1, Z=1)=$ $0,03 \cdot 0,85 \cdot 0,50 \cdot 0,71=0,0091$.

Entsprechend lassen sich alle Wahrscheinlichkeiten $p_{1}$ bis $p_{16}$ berechnen.
Die Tab. 2 hat dieselbe Form wie Tab. 2 in [8] (bzw. nach einer entsprechenden Einschränkung wie Tab. 3 in [8]), sodass alle Folgerungen zu Risiken und Exzessrisiken gültig bleiben, die aus Tab. 2 und 3 in [8] mathematisch abgeleitet wurden.

Diese Darstellung lässt sich erweitern, indem Wahrscheinlichkeiten zu einem deterministischen Ereignis hinzugemischt werden. Hierzu sei ein zweiter Versuchsaufbau mit anderer $\alpha$-Funktion realisiert, z. B.

$$
\alpha=20^{\circ}+X \cdot 40^{\circ}+Z \cdot 20^{\circ} .
$$

$\mathrm{Zu}$ diesem Versuchsaufbau 2 gehören ebenfalls 16 Responstypen mit Realisierungswahrscheinlichkeiten $q_{1}$ bis $q_{16}$.

Ein fester Anteil $a_{1}$ der im Ausgangszustand präparierten Photonen werde durch Versuchsaufbau 1 geschickt und alle anderen durch den Versuchsaufbau 2 (komplementärer Anteil $a_{2}=1-a_{1}$ ). Es sei determiniert, welches Photon durch Versuchsaufbau 1 läuft und welches durch Aufbau 2. Im Gesamtkollektiv der Photonen kommt der Responstyp $k$, $1 \leq k \leq 16$, mit Wahrscheinlichkeit

$$
w_{k}=a_{1} \cdot p_{k}+\left(1-a_{1}\right) \cdot q_{k}
$$

vor. Wiederum entsteht eine Tabelle wie - Tab. 2, aber mit Wahrscheinlichkeiten $w_{k}$ anstelle der Wahrscheinlichkeiten $p_{k}$. Auch in dieser erweiterten Situation bleiben alle Folgerungen zu Risiken und Exzessrisiken gültig, die aus Tab. 2 und 3 in Morfeld und Spallek [8] mathematisch abgeleitet wurden. Entscheidend ist allein, dass jedem der Responstypen eine definite Wahrscheinlichkeit zugeordnet werden kann, und die Typen als Ereignisse disjunkt sind. Einfache Summenformeln lassen sich auch nicht bei Annahme einer probabilistischen Responsvariablen rechtfertigen.

\section{Fazit}
- Die wesentlichen Aussagen in Mor- feld und Spallek [8] bleiben gültig, auch wenn
- die Expositionen intervallskaliert sind oder

\section{- der binäre Respons probabilistisch ist oder \\ - die Analysen auf Responstypen eingeschränkt werden, die in einem weiten oder engen Sinn adverse Effekte beschreiben. \\ - Wir stellen klar, dass Exzessrisiken und Differenzen von Exzessrisiken keine Wahrscheinlichkeiten im Sinne der Kolmogorov-Axiome darstellen.}

\section{Korrespondenzadresse}

\section{PD Dr. P. Morfeld}

Institut für Epidemiologie und Risikobewertung in der Arbeitswelt (IERA), Evonik Industries AG Rellinghauser Str. 1-11, 45128 Essen,

Deutschland

peter.morfeld@evonik.com

Danksagung. Wir danken Herrn Konietzka, UBA, für die Organisation des externen Reviews und den 3 Gutachtern (Prof. Baumgartner, Genf; Prof. Bartels, Bonn; Prof. Beisbart, Bern) für die intensive Durchsicht unserer Publikation [8], jedoch insbesondere für die sehr wertvollen Kommentare und Fragen. Wir danken einem unbekannten Reviewer für die intensive und akribische Durchsicht einer früheren Version dieses Manuskripts und für die äußerst hilfreichen Hinweise.

\section{Einhaltung ethischer Richtlinien}

Interessenkonflikt. P. Morfeld und M. Spallek geben an, dass kein Interessenkonflikt besteht.

Dieser Beitrag beinhaltet keine von den Autoren durchgeführten Studien an Menschen oder Tieren.

Open Access. This article is distributed under the terms of the Creative Commons Attribution 4.0 International License (http://creativecommons.org/ licenses/by/4.0/), which permits unrestricted use, distribution, and reproduction in any medium, provided you give appropriate credit to the original author(s) and the source, provide a link to the Creative Commons license, and indicate if changes were made.

\section{Literatur}

1. Audretsch J (2012) Die sonderbare Welt der Quanten: Eine Einführung, 2. Aufl. C.H.Beck, München

2. Audretsch J (2007) Entangled Systems - New Directions in Quantum Physics. Wiley-VCH, Weinheim

3. Bandelow C (1976) Einführung in die Wahrscheinlichkeitstheorie. Studienverlag Brockmeyer, Bochum

4. Becker P (2016) Berufskrankheiten - Auch von der Medizin zu beachtende rechtliche Vorgaben. Zentralbl Arbeitsmed Arbeitsschutz Ergon: doi:10.1007/s40664-016-0093-9 
5. Becker P (2015) Synkanzerogenese als Berufskrankheit. Zentralbl Arbeitsmed Arbeitsschutz Ergon 65(6):301-304

6. Bundesanstalt für Arbeitsschutz und Arbeitsmedizin (2014) TRGS 910 „Risikobezogenes Maßnahmenkonzept für Tätigkeiten mit krebserzeugenden Gefahrstoffen". http://www.baua.de/ de/Themen-von-A-Z/Gefahrstoffe/TRGS/TRGS910.html.Zugegriffen: 11. Febr. 2016

7. Fisz M (1976) Wahrscheinlichkeitsrechnung und mathematische Statistik. Deutscher Verlag der Wissenschaften, Berlin

8. Morfeld P, Spallek M (2015) Gefährdungsbeurteilung bei Exposition gegenüber mehreren krebserzeugenden Arbeitsstoffen. Zentralbl Arbeitsmed Arbeitsschutz Ergon 65(4):192-202

9. Morfeld P, Spallek M (2016) Synkanzerogenese ist keine Berufskrankheit. Zentralbl Arbeitsmed Arbeitsschutz Ergon:1-2. doi:http://dx.doi.org/10.1007/s40664-0160092-x

10. Schäcke G, HallierE(2004) Synkanzerogenese-die Position der Arbeitsmedizin. Zentralbl Arbeitsmed ArbeitsschutzErgon 54:146-158

\section{2,5 Millionen nosokomiale Infektionen jährlich in Europa}

In Europa sterben nach einer neu-

en Studie hochgerechnet mehr als

90.000 Patienten pro Jahr an Kran-

kenhausinfektionen.

Die Forscher um Alessandro Cassini vom Europäischen Zentrum für die Prävention und Kontrolle von Krankheiten (ECDC) gehen von mehr als 2,5 Millionen Infektionen aus, die sich Patienten erst in einer Klinik zuzogen. Für ihre Studie haben sie vor allem auf Daten dieses Zentrums zurückgegriffen. In die Auswertung flossen sechs häufige nosokomiale Infektionen ein, darunter Lungenentzündungen, Sepsis sowie Harnwegs- und Wundinfektionen.

Die Krankenhausinfektionen sind nach Angaben der Autoren zugleich Ursache für 2,5 Millionen beeinträchtigte oder verlorene Lebensjahre jährlich in Europa. Bei der völlig neuen Art der Berechnung sei auch berücksichtigt worden, dass solche Patienten immer Grunderkrankungen haben, betonte Mitautor Tim Eckmanns vom Robert Koch-Institut, das maßgeblich an der Studie beteiligt war. Die ermittelte Krankheitslast sei also nur auf die Krankenhausinfektionen zurückzuführen und nicht auf die ohnehin bei den Patienten vorhandenen Krankheiten. Basis für die Auswertung waren die Daten von rund 274.000 Patienten in rund 1.150 Akutkrankenhäusern, die in 30 europäischen Ländern 2011/12 erhoben worden waren. Nicht berücksichtigt wurden andere Einrichtungen wie zum Beispiel Reha-Zentren. Bei den Hochrechnungen wurden Krankenhausinfektionen, die durch multiresistente Erreger ausgelöst wurden, bewusst nicht separat ausgewiesen.

Quelle: Deutsches Ärzteblatt

basierend auf: Plos Medicine (2016)

DOI:10.1371/journal.pmed.1002150

\section{Weiterbildung Infektiologie wird gefördert}

Krankenhausinfektionen, multiresistente Erreger, Epidemien und neue Viren Fachwissen in Infektiologie wird dringend benötigt. Doch in Deutschland gibt es nur 300 Ärzte mit diesen Kenntnissen. Die Deutsche Gesellschaft für Infektiologie (DGI) schätzt den Bedarf auf mindestens 1000. Das Bundesgesundheitsministerium fördert deshalb infektiologische Zusatzweiterbildungen mit je 30.000 Euro. Die Förderung ist im Krankenhausentgeltgesetz festgeschrieben und gilt seit dem 1. Januar 2016. Sie greift bei Weiterbildungen, die in den Jahren 2016 bis 2019 beginnen. Wie entscheidend sich das Fachwissen eines Infektiologen auf Behandlungsergebnisse auswirkt, zeige beispielhaft eine aktuelle Auswertung mehrerer Studien. Behandelt ein spezialisierter Infektiologe eine durch das Bakterium Staphylococcus aureus ausgelöste Sepsis, sinke die Sterblichkeit der Patienten um rund die Hälfte im Vergleich zum üblichen Ärzteteam. Seit 2015 bieten die Deutsche Gesellschaft für Innere Medizin (DGIM) und die DGI gemeinsame Antibiotic-Stewardship (ABS)Kurse für Internisten an. Darin schulen Experten den professionellen Einsatz von Antibiotika in der Klinik. Die Kurse werden von der Akademie für Infektionsmedizin durchgeführt und von der DGIM bezuschusst.

\author{
Quelle: Arbeitsgemeinschaft der \\ Wissenschaftlichen Medizinischen \\ Fachgesellschaften, \\ www.dgi-net.de, \\ www.dgim.de
}

